\title{
Structured waves near the plasma frequency observed in three auroral rocket flights
}

\author{
M. Samara ${ }^{1,2}$ and J. LaBelle ${ }^{1}$ \\ ${ }^{1}$ Dartmouth College, Department of Physics and Astronomy, Hanover, NH, USA \\ ${ }^{2}$ now at: Southwest Research Institute, San Antonio, TX, USA
}

Received: 2 September 2005 - Revised: 19 April 2006 - Accepted: 29 August 2006 - Published: 21 November 2006

\begin{abstract}
We present observations of waves at and just above the plasma frequency $\left(f_{p e}\right)$ from three high frequency electric field experiments on three recent rockets launched to altitudes of $300-900 \mathrm{~km}$ in active aurora. The predominant observed HF waves just above $f_{p e}$ are narrowband, shortlived emissions with amplitudes ranging from $<1 \mathrm{mV} / \mathrm{m}$ to $20 \mathrm{mV} / \mathrm{m}$, often associated with structured electron density. The nature of these HF waves, as determined from frequency-time spectrograms, is highly variable: in some cases, the frequency decreases monotonically with time as in the "HF-chirps" previously reported (McAdams and LaBelle, 1999), but in other cases rising frequencies are observed, or features which alternately rise and fall in frequency. They exhibit two timescales of amplitude variation: a short timescale, typically $50-100 \mathrm{~ms}$, associated with individual discrete features, and a longer timescale associated with the general decrease in the amplitudes of the emissions as the rocket moves away from where the condition $f \sim f_{p e}$ holds. The latter timescale ranges from 0.6 to $6.0 \mathrm{~s}$, corresponding to distances of $2-7 \mathrm{~km}$, assuming the phenomenon to be stationary and using the rocket velocity to convert time to distance.
\end{abstract}

Keywords. Ionosphere (Auroral ionosphere; Plasma waves and instabilities) - Space plasma physics (Waves and instabilities)

\section{Introduction}

Many previous space plasma experiments have included continuous resolution full waveform measurements of Langmuir waves. For example, (Gurnett et al., 1981) presented observations of the parametric decay and spatial collapse of Langmuir waves in the solar wind setting. However, be-

Correspondence to: M. Samara

(msamara@swri.edu) cause of the higher frequency, $\mathrm{MHz}$ instead of $\mathrm{kHz}$, Langmuir wave waveform observations in the ionosphere were limited for a long time to envelope measurements (for example, Boehm, 1987; Ergun et al., 1991a,b) or to the waveformcapture technique (Kintner et al., 1995). Recently McAdams and LaBelle (1999) flew a continuous waveform receiver on a sounding rocket taking measurements in the auroral ionosphere. They observed the fine structure of auroral Langmuir waves near the plasma frequency, finding distinctive frequency structure in two different regions. "HF-Chirps", extremely narrowband and short lived emissions, were observed at lower altitudes when $f_{p e}>f_{c e}$. "Bands", also narrowband but lasting longer, were observed at higher altitudes when $f_{p e}<f_{c e}$. Samara (2005) reports a statistical analysis of the bursty Langmuir waves associated with the bands.

McAdams and LaBelle (1999) labeled as "HF-Chirps" the emissions they observed just above the plasma frequency, about $2 \mathrm{MHz}$ in their experiment, in the overdense plasma where $f_{p e}>f_{c e}$. The chirps were associated with electron density irregularities with scales sizes meters to a kilometer or so. Their bandwidth is $300-600 \mathrm{~Hz}$, their amplitudes are $\sim 0.7 \mathrm{mV} / \mathrm{m}$, and they last about $100 \mathrm{~ms}$. They often appear in pairs or multiplets. A distinctive characteristic is that their frequency decreases with time, at a typical rate of $50 \mathrm{kHz} / \mathrm{s}$. This tendency of their frequency to decrease inspired the name "chirps." In a later paper, McAdams et al. (2000) put forth a model of the HF "chirp" emissions as quasitrapped eigenmodes in a field aligned density depletion, where the escaping waves retain the frequency structure of the eigenmodes. This was quantitatively consistent with the observed frequency spacings of HF "chirp" emissions $(0.1-4 \mathrm{kHz})$, the fact that they are equally spaced, as well as the number of modes predicted for a given density cavity. More recently, Yoon and LaBelle (2005) developed a theoretical treatment of Langmuir wave eigenmodes based on a Lorentzian profile of density irregularity and using nonlocal kinetic theory wave equations.

Published by Copernicus GmbH on behalf of the European Geosciences Union. 
Table 1. Launch details for each rocket. $L_{e f f}$ is the effective antenna length.

\begin{tabular}{cccccc}
\hline Rocket & Launch & Apogee & $L_{\text {eff }}$ & Spin & Orientation \\
\hline PHAZE II & 10 Feb. 97 & $945 \mathrm{~km} @ 556 \mathrm{~s}$ & $0.3 \mathrm{~m}$ & $0.6 \mathrm{~Hz}$ & $\|$ to B \\
SIERRA & 14 Jan. 02 & $735 \mathrm{~km} @ 500 \mathrm{~s}$ & $3 \mathrm{~m}$ & $0.3 \mathrm{~Hz}$ & cartwheel \\
RACE & 6 Feb. 02 & $922 \mathrm{~km} @ 550 \mathrm{~s}$ & $0.3 \mathrm{~m}$ & $0.95 \mathrm{~Hz}$ & $\|$ to B \\
\hline
\end{tabular}

Table 2. Capacitances and correction factors for each rocket for the regions analyzed in this paper. The input capacitance was directly measured for the RACE and PHAZE II experiments, but in the SIERRA experiment, it was estimated from a theoretical model.

\begin{tabular}{cccc}
\hline Rocket & $C_{i n}(\mathrm{pF})$ & $C_{\text {sheath }}(\mathrm{pF})$ & factor \\
\hline PHAZE II & 12.5 & $4.32-5.14$ & $3.89-3.43$ \\
RACE & 30.8 & $10.755-11.85$ & $3.86-3.6$ \\
SIERRA & 40.28 & $311.486-371.3$ & $1.13-1.108$ \\
\hline
\end{tabular}

In 2002, two sounding rockets including HF waveform receivers were launched from Poker Flat, Alaska, to altitudes of 700-950 km in active aurora, providing additional opportunities to detect and study structure of auroral Langmuir waves. Both experiments detected narrowband, short-lived emissions just above $f_{p e}$ that are similar to the HF-chirps in some ways but exhibit significant differences. In this paper, we document the Langmuir wave signatures observed in the 2002 experiments and parametrize the spatial decay of the "chirp" signals outside of their source regions. Section 2 presents instrument details, and Sect. 3 presents data of the observations made with the three rockets and compares them quantitatively.

\section{Instrumentation}

The dataset we are considering here consists of three HF electric field experiments on three recent rockets. All three used either double-probe or wire-type antennas to detect the electric field of the waves. Table 1 gives information such as apogee and antenna length for each rocket, since these are important for interpreting and comparing the observations.

Physics of Auroral Zone Electrons (PHAZE II) was launched from Poker Flat on 10 February 1997, at 05:17 UT reaching an apogee of $945.3 \mathrm{~km}$ at about $556 \mathrm{~s}$. The spin axis was parallel to the background magnetic field, and the electric field double-probe antenna consisted of $\sim 4.5 \mathrm{~cm}$ diameter cylindrical probes separated by $0.3 \mathrm{~m}$ along an axial boom mounted forward of the payload; i.e., the sensor detected the component of electric field parallel to the magnetic field.
Sounding the Ion Energization Region-Resolving Ambiguities (SIERRA) was launched from Poker Flat on 14 January 2002 at 08:23 UT reaching an apogee of $735 \mathrm{~km}$ at about $500 \mathrm{~s}$. In this experiment, the spin axis was perpendicular to the magnetic field (cartwheel mode). The electric field sensor was in effect a wire dipole, consisting of the conducting Weitzmann boom stacer elements, each having physical length of approximately $3 \mathrm{~m}$ and average diameter of $2 \mathrm{~cm}$.

The Rocket Auroral Correlator Experiment (RACE) was launched from Poker Flat on 6 February 2002 at 09:38 UT reaching an apogee of $922 \mathrm{~km}$ at about $550 \mathrm{~s}$. The rocket spin axis was aligned with the background magnetic field. The electric field sensor was a double-probe with separation $0.3 \mathrm{~m}$ and orientation parallel to the magnetic field, identical to the sensor on PHAZE II described above, except that the probes in the case of RACE were spherical and approximately $6 \mathrm{~cm}$ diameter.

All three payloads included similar HF receivers. The HF receiver on these rockets measured continuously the full electric field waveform below $5 \mathrm{MHz}$, which was transmitted to the ground station on an analog telemetry channel. Due to the limited dynamic range of the analog telemetry, it was necessary to use automatic gain control (AGC) to ensure that the transmitter was always fully modulated. To prevent LF signals such as auroral hiss from controlling the receiver gain, the received signals were passed through a high pass-filter, set at $400 \mathrm{kHz}$ on PHAZE II and $100 \mathrm{kHz}$ on SIERRA and RACE. At the ground station, the signals were recorded on DTR-6/8/16 tape drives and digitized on replay, at a 5 Msamples/s rate for PHAZE II and 10 Msamples/s for SIERRA and RACE.

In all three cases, folding in the AGC yields the detected probe-to-probe voltage at the receiver input. Conversion to electric field requires dividing by an effective antenna length, assumed to be the probe separation in the case of the doubleprobe sensors and half of the physical length in the case of the wire-type antenna. It is also essential to account for the reduction in signal due to the finite input capacitance of the pre-amplifier and the sheath capacitance of the probes. The desired voltage is $V_{\text {plasma }}$, but $V_{\text {measured }}$ is actually measured at the probe. The input capacitance of the preamp and the probe sheath capacitance are in effect a capacitor-divider circuit, and the measured voltage must be corrected upward by a factor of $\left(C_{i n}+C_{\text {sheath }}\right) / C_{\text {sheath }}$ to obtain the desired voltage $V_{\text {plasma. }}$. 

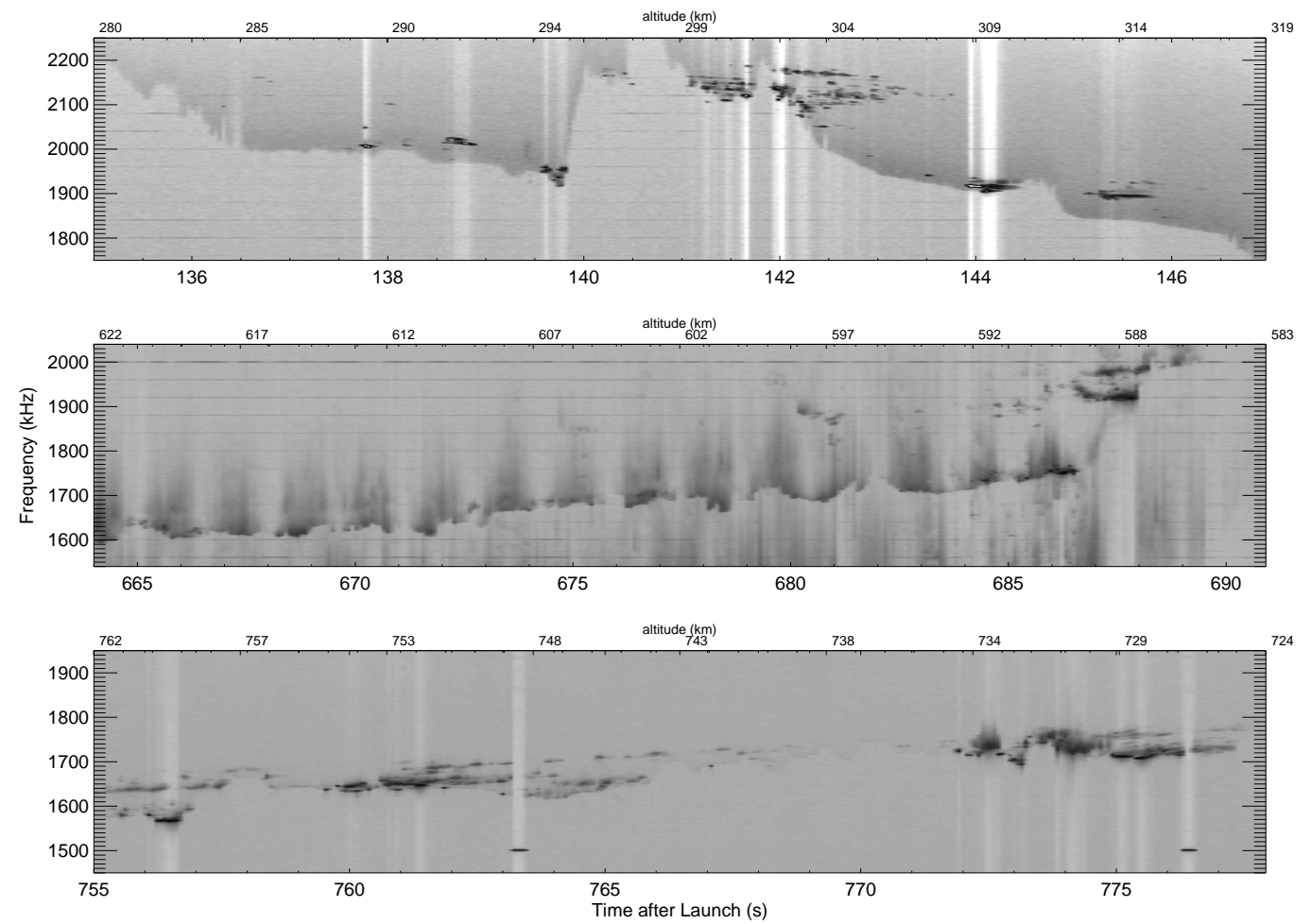

Fig. 1. Electric field spectrograms of selected intervals on three rocket flights: PHAZE II (top panel), SIERRA (middle panel), and RACE (bottom panel). The grayscale dynamic range is $40 \mathrm{~dB}$ for PHAZE II, $50 \mathrm{~dB}$ for SIERRA, and $70 \mathrm{~dB}$ for RACE. The time interval shown is $11 \mathrm{~s}$ for PHAZE II and $27-28 \mathrm{~s}$ for the other two flights, but the range of altitudes is about the same, $\sim 38 \mathrm{~km}$, in all three panels. PHAZE II was at a much lower absolute altitude than were the other two flights. AGC was not folded in, so vertical light bands correspond to times when intense wave activity caused the AGC to reduce the gain; intense bands at $1500 \mathrm{kHz}$ at 763 and $776 \mathrm{~s}$ in the bottom panel are artificial calibration signals. In all three panels, a lower cutoff of the waves corresponds to the plasma frequency (ranging from $2200 \mathrm{kHz}$ to $1800 \mathrm{kHz}$ in the top panel, from 1800 to $2000 \mathrm{kHz}$ in the middle panel, and from 1550 to $1700 \mathrm{kHz}$ in the bottom panel). Structured Langmuir waves occur at $f \geq f_{p e}$ and often coincide with regions of electron density structures and gradients.

The input capacitances, measured in the field for PHAZE II and RACE, were $12.5 \mathrm{pF}$ (PHAZE II) and $30.8 \mathrm{pF}$ (RACE), as given in Table 2. For SIERRA, it was impossible to directly measure $C_{i n}$ because the antenna was never in flight configuration before the actual flight. We therefore estimated $C_{i n}$ using a parallel plate model of the boom mounting hardware (approximately $7.1 \mathrm{~cm}$ by $18.8 \mathrm{~cm}$ with $0.32 \mathrm{~cm}$ separation) in parallel with the measured pre-amplifier input, implying $C_{i n}=40.28 \mathrm{pF}$ as shown in Table 2. Assuming $T_{e}$ of order $0.1 \mathrm{eV}$ and using the measured electron densities for the periods analyzed below $\left(42000-6500 \mathrm{~cm}^{-3}\right.$ in the case of PHAZE II, $31000-40000 \mathrm{~cm}^{-3}$ in the case of RACE, and $32000-50000 \mathrm{~cm}^{-3}$ in the case of SIERRA), the appropriate Debye lengths are $1.15-0.92 \mathrm{~cm}$ (PHAZE II), $1.34-1.17 \mathrm{~cm}$ (RACE) and $1.32-1.1 \mathrm{~cm}$ (SIERRA). Assuming a sheath approximately one Debye length thick implies a sheath capacitance of 4.32-5.14 pF for the PHAZE II cylindrical probes, $10.76-11.85 \mathrm{pF}$ for the RACE spherical probes and $311.49-371.34 \mathrm{pF}$ for the SIERRA cylindrical dipole element. Thus, the resulting voltage-correction factors are
3.89-3.43 for PHAZE II, 3.86-3.6 for RACE and 1.13-1.11 for SIERRA. Table 2 summarizes these results.

\section{Data presentation}

Figure 1 shows an overview of selected interesting regions encountered by the three flights. All three panels consist of grayscale displays of sequences of Fast Fourier Transform (FFT) spectra of the measured HF electric field. The FFT length is 4096 samples (PHAZE II data), 8192 samples (SIERRA data) and 8192 samples (RACE data), implying a time interval per FFT of about $0.8 \mathrm{~s}$ and a frequency resolution of $1.2 \mathrm{kHz}$ in all three cases. (The sample rate in the PHAZE II experiment was half than in the other two experiments.) The FFTs have been averaged by a factor of 49 (PHAZE II data), 110 (RACE data) and 94 (SIERRA data) in order to arrange that the total altitude range represented in each panel is approximately the same in each case, $\approx 38 \mathrm{~km}$ : the PHAZE II observations correspond to the upleg at 280$318.5 \mathrm{~km}$, and those of SIERRA and RACE correspond to the 

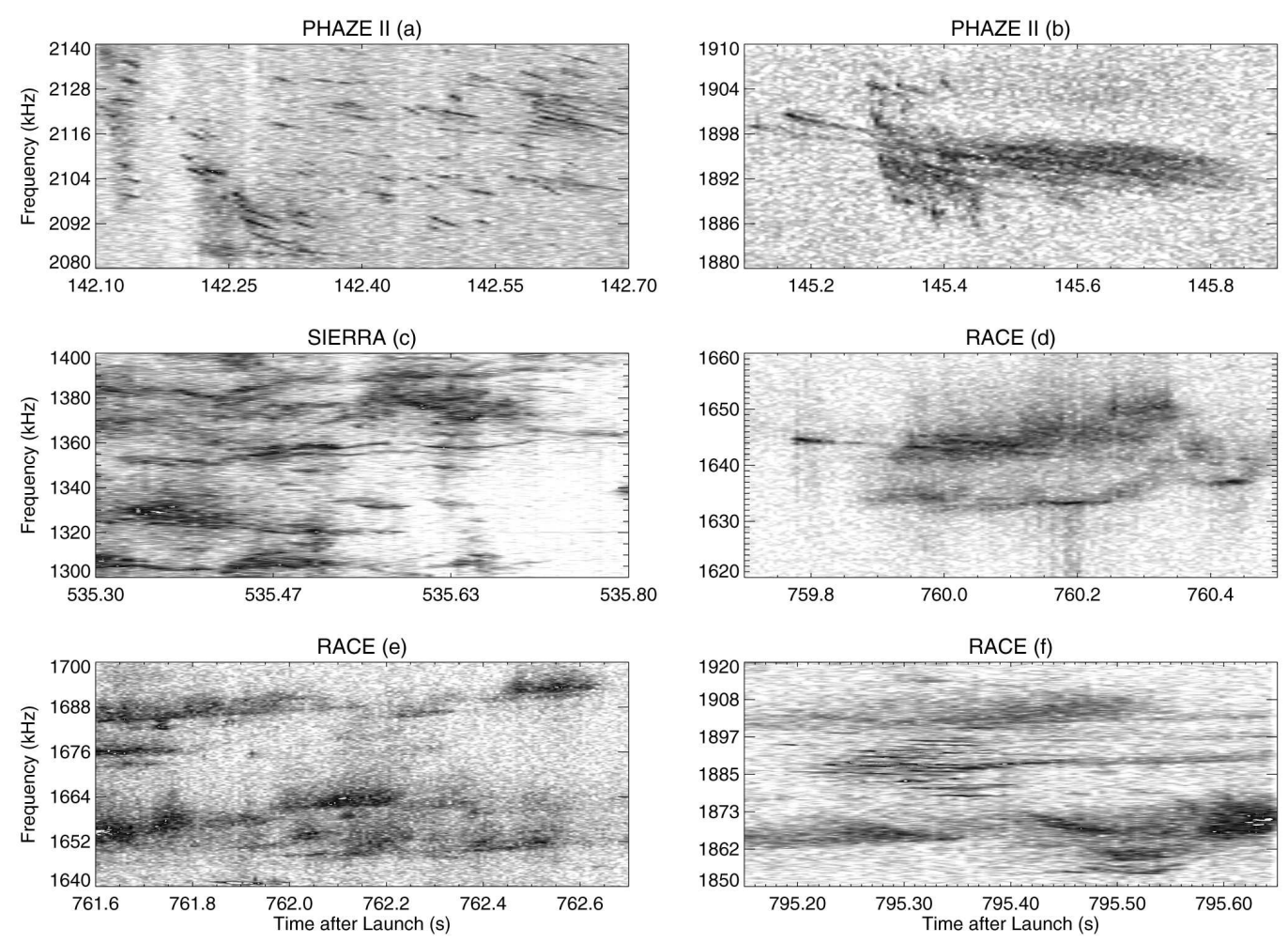

Fig. 2. Detailed electric field spectrograms showing the structure of the "HF-chirp" emissions observed just above $f_{p e}$ on three flights: (a) and (b) are from PHAZE II, (c) from SIERRA and (d), (e) and (f) from RACE. There is a $40 \mathrm{db}$ (panels b, c, e, f), $50 \mathrm{db}$ (a) and $60 \mathrm{~dB}$ (d) difference between white and black on the grayscales. The AGC has not been accounted for in these displays. While the fine structures observed with PHAZE II all tend to decrease in frequency with time, inspiring the name "HF-chirps," those observed on the other two rocket flights exhibit both decreasing and increasing frequencies.

downleg at altitudes 621.5 to $582.75 \mathrm{~km}$ and 762 to $724 \mathrm{~km}$, respectively. Hence, the AGC and filter are not folded in for these panels. The light vertical regions (for example, around $144 \mathrm{~s}$ on PHAZE II, $686 \mathrm{~s}$ on SIERRA and $755 \mathrm{~s}$ on RACE) result from the correction of the AGC when large signals are encountered and the gain is reduced. In the third panel, the two horizontal lines at about $1500 \mathrm{kHz}$, shortly after 763 and $776 \mathrm{~s}$, are artificially injected calibration signals.

The cutoff at $f_{p e}$ provides a means of determining plasma density. In all three panels the $f_{p e}$ cutoff is clear; it ranges from 1840 to $2300 \mathrm{kHz}$ for PHAZEII, 1600 to $2000 \mathrm{kHz}$ for SIERRA and 1570 to $1800 \mathrm{kHz}$ for RACE. The local $f_{c e}$ is about $1357 \mathrm{kHz}$ (PHAZE II), $1224 \mathrm{kHz}$ (SIERRA) and $1152 \mathrm{kHz}$ (RACE). Hence, all three observations correspond to overdense plasma conditions, where $f_{p e}>f_{c e}$. In all three flights intense waves occur at and just above $f_{p e}$ and exhibit fine frequency structure with bandwidths of a few $\mathrm{kHz}$ or less. The structured waves are associated with regions of enhanced electron density and associated density irregularities with spatial scales of meters to kilometers and amplitudes of order 10 percent. Because of the limited number of high frequency antennas and receivers deployed on these flights, only one antenna in the case of SIERRA and PHAZE II, the wave mode cannot be identified with certainty. Two modes which occur in this frequency range just above $f_{p e}$ for $f_{p e}>f_{c e}$ are the LO-mode, which has a cutoff at $f_{p e}$, and the Langmuirupper hybrid modes, which have a resonance at $f_{p e}$ for parallel propagation (k-vector along the magnetic field). The concentration of the wave power at $f_{p e}$ suggests that these are Langmuir waves, although it is possible that $\mathrm{LO}$ waves, upon reflection at $f=f_{p e}$, can have enhanced wave energy density at the reflection point where the group velocity gets slow. At other times on the PHAZE II flight, much weaker signals appear near $f_{p e}$ which McAdams and LaBelle (1999) interpret as LO mode. However, the greater maximum amplitude of the waves in Fig. 1 argues for the Langmuir mode interpretation. The spin dependence observed in the SIERRA data, whereby the signal intensity drops out when the antenna is nearly perpendicular to the magnetic field, also supports the interpretation of these signals as parallel-propagating Langmuir modes, which would have their electric field along the background magnetic field. Despite some uncertainty in the mode identification, these structured, time-variable waves observed at and just above $f_{p e}$ are hereinafter referred to as Langmuir waves. 


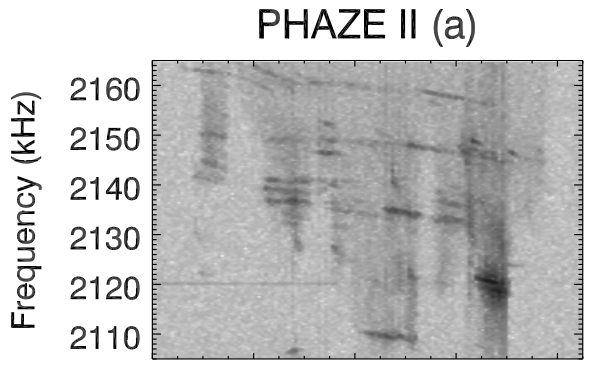

$\begin{array}{lllll}141.0 & 141.2 & 141.4 & 141.6 & 141.8\end{array}$

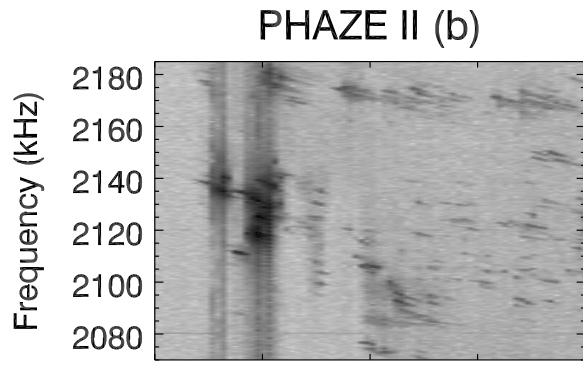

141.85142 .23142 .60142 .98143 .35

$\log _{10}\left[V^{2} /\left(m^{2} \mathrm{~Hz}\right)\right]$
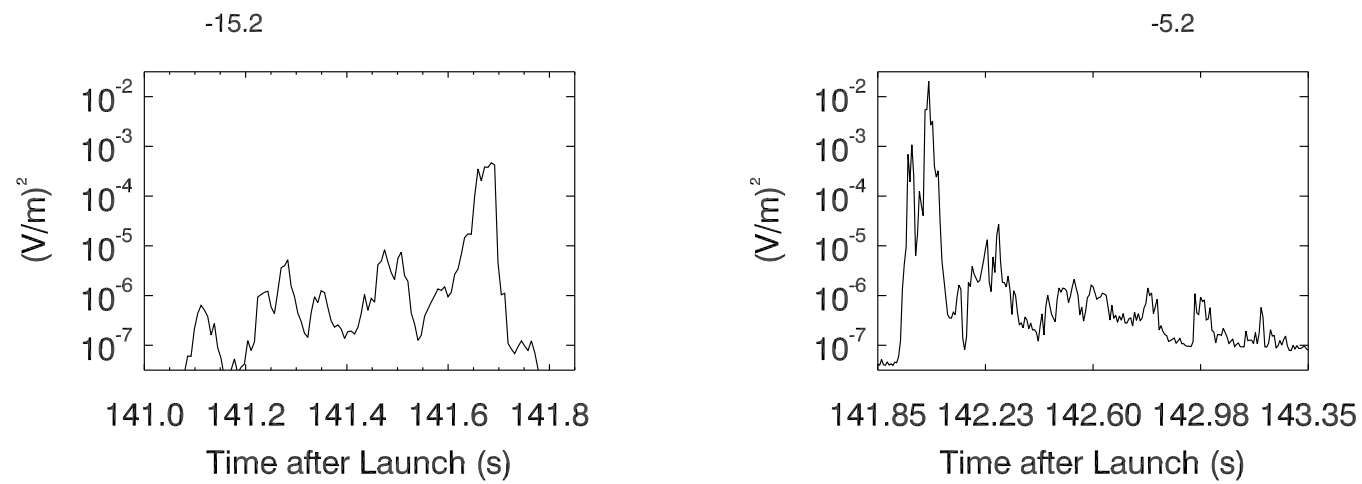

Fig. 3. Top panels show electric field spectrograms of selected short intervals of Langmuir wave fine structures for (a) 141.00-141.85 s and (b) 141.85-143.35 s during the PHAZE II rocket flight, corresponding to $300 \mathrm{~km}$ and $304 \mathrm{~km}$, respectively. The bottom panels show wave power integrated over the entire frequency range displayed in the top panels. The wave power is largest when the rocket is nearest to the presumed source region, where the wave frequency is closest to local $f_{p e}$, and away from that condition, the wave power decreases.

The wave intensities determined from examination of individual spectra imply amplitudes in the range of 0.76 to $22 \mathrm{mV} / \mathrm{m}$ for PHAZE II, 0.02 to $0.06 \mathrm{mV} / \mathrm{m}$ for SIERRA and 0.12 to $1.2 \mathrm{mV} / \mathrm{m}$ for RACE. These Langmuir waves are much weaker than in some of the cases in the literature, such as, for example, $50 \mathrm{mV} / \mathrm{m}$ Langmuir waves reported by Ergun et al. (1991a). The far smaller amplitudes inferred for the SIERRA experiment warrant further discussion. The physical antenna length on SIERRA was $6 \mathrm{~m}$ versus $30 \mathrm{~cm}$ on the other two flights. Langmuir waves generally have short wavelengths, on the order of $\lambda \sim 1 \mathrm{~m}$, in order to be resonant with the relatively low energy auroral electrons. For wavelengths less than the antenna length $(L)$, the antenna response falls off significantly, theoretically something like $\sin (L / \lambda)$ for double-probe antennas, as discussed e.g. by Temerin (1978). In the cases of RACE and PHAZE II, the antenna is almost certainly smaller than the wavelength, so there is littler or no correction, but in the case of SIERRA, the wave amplitude may be considerably underestimated. For example, for a plausible value $L / \lambda \sim 6$, the correction factor is of order 10-20, which would make the amplitudes observed in the SIERRA experiment comparable to those observed in the RACE experiment and in the range of those observed in the PHAZE II experiment. (Because the correction of the amplitudes based on the wavelength of Langmuir waves brings the SIERRA data into agreement with those on the other two flights could be taken as evidence supporting the identification of the waves as Langmuir mode.) The low amplitudes inferred above in the SIERRA case should therefore not be interpreted as the wave electric field strength.

Figure 2 shows an "atlas" of wave fine structure observations giving an impression of the variety of wave fine structures observed during each flight. Each panel is an electric field spectrogram similar to Fig. 1, except with higher time resolution. As in Fig. 1, in order to better show the wave structure within the limited grayscale dynamic range, the AGC and high-pass filter are not folded in for these displays.

The PHAZE II examples shown in Figs. 2a, b were observed on the upleg in the altitude range 290 to $315 \mathrm{~km}$. They are dominated by descending tones, called "chirps" by McAdams and LaBelle (1999), which have average downward slopes of $50 \mathrm{kHz} / \mathrm{s}$, ranging from 0 to $80 \mathrm{kHz} / \mathrm{s}$. Typical duration is $50 \mathrm{~ms}$, ranging from 10 to $300 \mathrm{~ms}$ (see Fig. 2c of McAdams and LaBelle, 1999). McAdams and LaBelle (1999) report no correlation with the measured local electron 


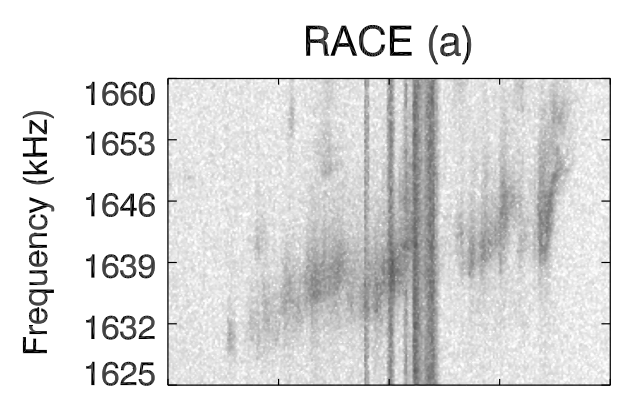

754.60755 .45756 .30757 .15758 .00
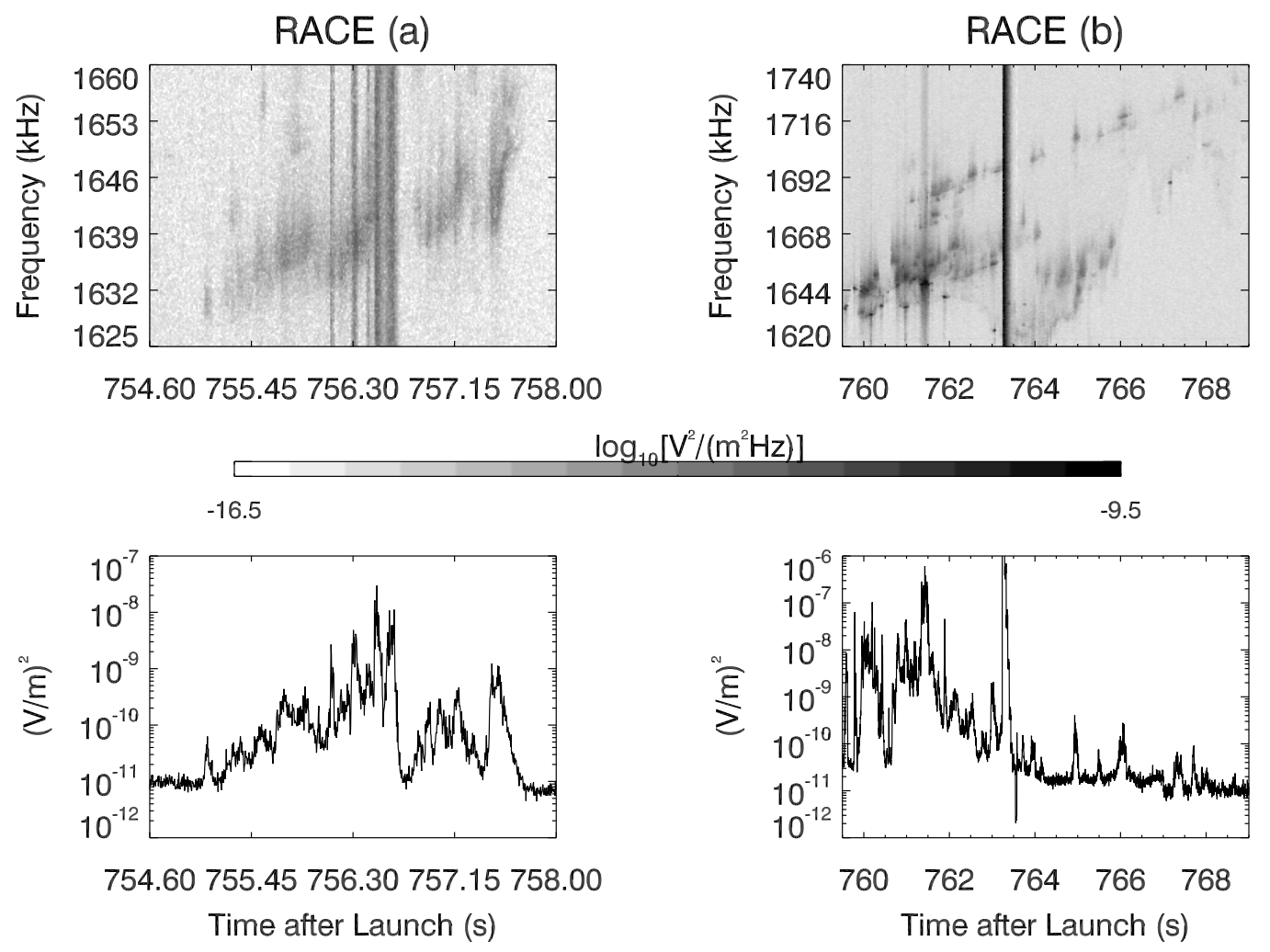

Fig. 4. Top panels show electric field spectrograms of selected short intervals of Langmuir wave fine structures for (a) 754.6-758.0 s and (b) $759.0-768.5 \mathrm{~s}$ during the RACE rocket flight, corresponding to $\sim 761 \mathrm{~km}$ and $\sim 748 \mathrm{~km}$, respectively. The bottom panels show wave power integrated over portions of the frequency ranges displayed in the top panels.

distribution function. However, such correlations can be subtle and notoriously difficult to detect, since the observed particle distribution function, reflecting the presence of the waves, is usually plateaued out and rarely exhibits the unstable feature giving rise to the waves, especially if the distribution function measurement is taken over a time interval longer than a few tens of ms. Previous studies, even those which have been successful in identifying features of the electron distribution function associated with Langmuir waves, show the difficulty in recognizing these (e.g., McFadden et al., 1986). Hence, the absence of such features does not comprise proof that the waves are not Langmuir mode.

The examples of HF wave structure observed in the SIERRA and RACE experiments sometimes included downward slopes, but they are not the dominant feature. Instead, these observations show both upward and downward slopes, with more upward than downward, in the case of SIERRA (Fig. 2c), and mostly upward slopes in the case of RACE (Fig. 2d, e, f). Figure 2e shows examples of the mostly upward slopes observed during the RACE experiment. In the SIERRA observations (Fig. 2c) "bird-like" sturctures occur, which exhibit both upward and downward slopes, such as that at $1350 \mathrm{kHz}$ from 535.45 to $535.55 \mathrm{~s}$, as well as structures which decrease monotonically with time, such as the one between 1315 and $1325 \mathrm{kHz}$ from 535.4 to $535.5 \mathrm{~s}$. In the case of SIERRA the observed features had an average upward slope of $20 \mathrm{kHz} / \mathrm{s}$, ranging from 0 to $30 \mathrm{kHz} / \mathrm{s}$. Typical duration is $70 \mathrm{~ms}$, ranging from 10 to $100 \mathrm{~ms}$. In the case of the RACE observations the average upward slope is $20 \mathrm{kHz} / \mathrm{s}$, ranging from 0 to $50 \mathrm{kHz} / \mathrm{s}$. Typical duration is $100 \mathrm{~ms}$, ranging from 10 to $200 \mathrm{~ms}$.

As noted above and by McAdams and LaBelle (1999) based on PHAZE II data, these structured Langmuir wave emissions occur in regions characterized by electron density irregularities, with the tendency to get the most intense emissions when the emission frequency $(f)$ is near the local $f_{p e}$. There are two timescales associated with that decay of the Langmuir wave fine structures: the average $50-100 \mathrm{~ms}$ lifetime of the individual "HF-chirp" features, noted above; and the longer lifetime associated with groups of them. For the former timescale, McAdams and LaBelle (1999) (see Fig. 2.) reported typical durations of individual "HF-chirps" to be $100 \mathrm{~ms}$ in the PHAZE II case, and from SIERRA and RACE observations, we measure similar average lifetimes of about $70 \mathrm{~ms}$ and $100 \mathrm{~ms}$ respectively.

We now turn to measurements of decay times of groups of "HF-chirp" features. The top left-hand panel of Fig. 3 shows a spectrogram display of an example of structured Langmuir 
waves observed in the PHAZE II experiment at 141.00$141.85 \mathrm{~s}$, corresponding to $300 \mathrm{~km}$ altitude. The AGC has been folded into this spectrogram to provide an accurate depiction of wave amplitudes; dark vertical lines result from the action of the AGC, whereby the intense signals received force the AGC into a low gain state and thereby lower the instument signal-to-noise ratio. The bottom left-hand panel shows the wave power in units of $(\mathrm{V} / \mathrm{m})^{2}$, integrated over the entire $60-\mathrm{kHz}$ frequency range $(2105-2165 \mathrm{kHz})$ shown in the top panel. These plots clearly show a decrease in wave power between the presumed source of the waves, near about $141.65-141.70 \mathrm{~s}$, where $f \approx f_{p e}$ and the wave power maximizes, and earlier times where the emissions lie at frequencies above $f_{p e}$ and have wave powers a factor of 30-300 lower. In this case, the waves lie at offsets from $0-60 \mathrm{kHz}$ (about 3\%) above local $f_{p e}$. The decay time $(\tau)$ may be estimated by fitting an exponential decay to these plots, and extracting the time that corresponds to a change in the integrated power spectral density of $1 / e$. From the example of Fig. 3a we obtain from this method a decay time $(\tau)$ of about $0.6 \mathrm{~s}$. If the wave decay is viewed as spatial rather than temporal, and the rocket velocity is used to convert the decay time to a distance, then $0.6 \mathrm{~s}$ corresponds to a decay distance of $2.3 \mathrm{~km}$.

The right hand panels of Fig. 3 show data from another group of "HF-chirp" emissions observed a few seconds later on the same PHAZE II flight, at $141.85-143.35 \mathrm{~s}$, corresponding to $304 \mathrm{~km}$ altitude; the bottom right-hand panel again shows wave power integrated over the entire frequency band displayed in the spectrogram (Fig. 3), in this case 2075$2182 \mathrm{kHz}$. This second example appears similar to the first: the most intense waves occur at $142.00 \mathrm{~s}$, and the wave power decreases as the rocket moves away from that region. In this case, the waves become offset from local $f_{p e}$ by more than $100 \mathrm{kHz}$ (about 5\%). For this example, we infer a decay time of $\tau \sim 1.15 \mathrm{~s}$. If, as above, the decay is interpreted as spatial rather than temporal, the decay distance would be $3.8 \mathrm{~km}$.

Figure 4 shows similar observations from the RACE experiment. The left-hand panels (Fig. 4a) correspond to $754.6-758.0 \mathrm{~s}$, or an altitude of $761 \mathrm{~km}$. As explained above, dark vertical features result from the AGC action. The bottom left-hand panel shows the wave power integrated over the entire $35-\mathrm{kHz}$ frequency range displayed in the spectrogram $(1625-1660 \mathrm{kHz})$. The most intense waves occur at about $756.5 \mathrm{~s}$, and the wave power decreases at earlier times. In this case the signals remain within about $30 \mathrm{kHz}$ (about $2 \%$ ) of local $f_{p e}$. Using the method described above, we infer a wave decay time of $\tau \sim 1.6 \mathrm{~s}$ for the interval 754.7$756.5 \mathrm{~s}$, which corresponds to $1.3 \mathrm{~km}$ if the decay is spatial rather than temporal. The right-hand panels (Fig. 4b) correspond to $759.0-768.5 \mathrm{~s}$, corresponding to $745-752 \mathrm{~km}$ in altitude. The vertical dark lines at, for example, $761.5 \mathrm{~s}$, result from action of the AGC, but the vertical line at $763.6 \mathrm{~s}$ and the corresponding peak in wave power in the lower right panel are due to an artificially injected calibration signal.
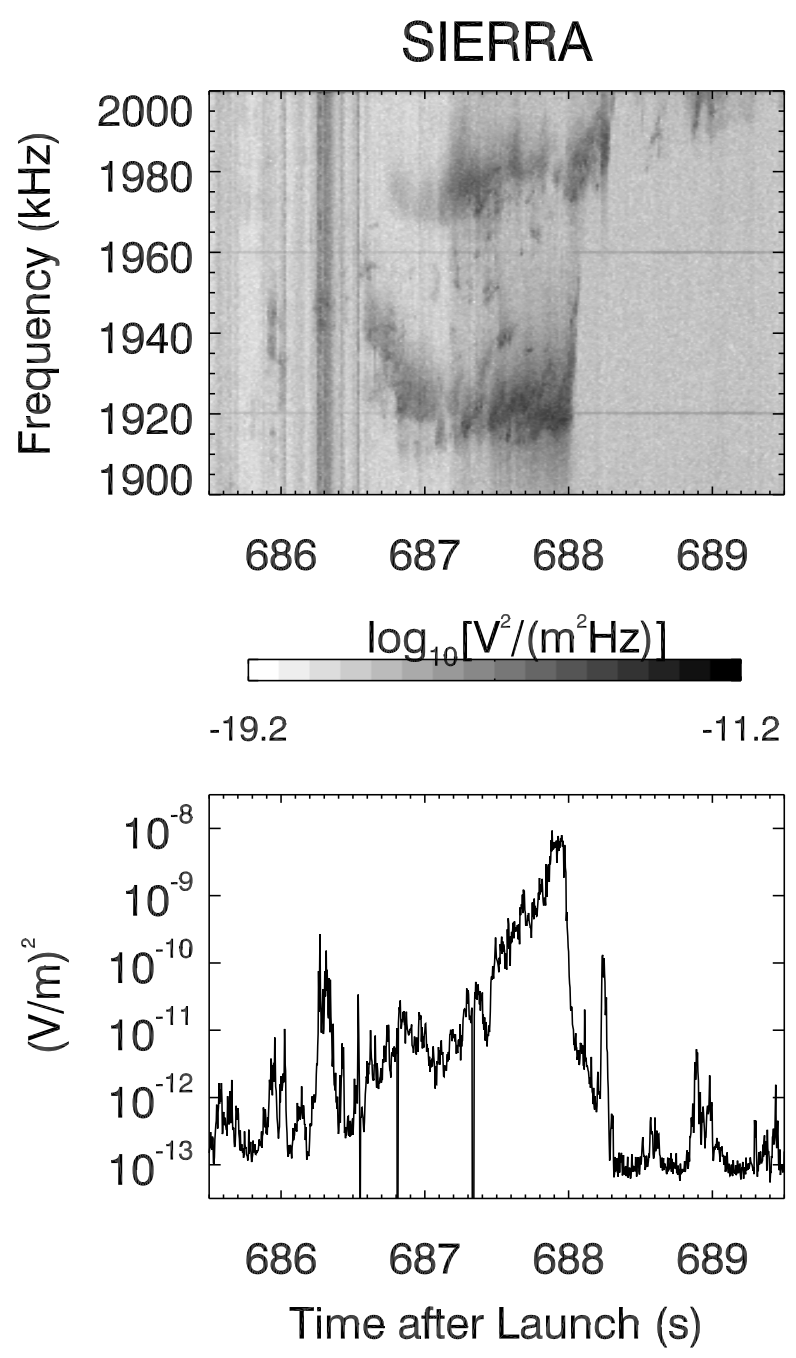

Fig. 5. (a) Electric field spectrogram recorded for 685.5-689.5 s during the SIERRA rocket flight, corresponding to $585-591 \mathrm{~km}$; (b) Wave power integrated over the entire $100-\mathrm{kHz}$ displayed frequency band.

The bottom right panel again shows wave power in units of $(\mathrm{V} / \mathrm{m})^{2}$, but in this case the power is integrated not over the entire $1620-1740 \mathrm{kHz}$ frequency range but over a frequency band which tracks the emission, increasing in frequency with time, starting at $1620-1740 \mathrm{kHz}$ and ending at $1704-1740 \mathrm{kHz}$. In this case the waves are offset from local $f_{p e}$ by up to $\sim 90 \mathrm{kHz}(5.6 \%)$. The characteristic decrease in wave power away from the presumed source region where $f \approx f_{p e}$ is clear in this plot; the wave decay time inferred from fitting an exponential decay to the data is $\tau \sim 6.5 \mathrm{~s}$. If interpreted as spatial rather than temporal, and using the rocket velocity to convert from time to distance, this decay time corresponds to a decay distance of $7 \mathrm{~km}$.

Finally, Fig. 5 shows a structured Langmuir wave event detected with the HF wave receiver on the SIERRA rocket 
Table 3. Summary of relevant characteristics of the waves. The decay distance is calculated assuming that the decaying waves are spatial structures.

\begin{tabular}{ccccccc}
\hline Rocket & chirp lifetime & intensity & slope & slope dir. & decay time & decay dist. \\
\hline PHAZE II & $50 \mathrm{~ms}$ & $0.72-2.2 \mathrm{mV} / \mathrm{m}$ & $50 \mathrm{kHz} / \mathrm{s}$ & down & $0.6-1.15 \mathrm{~s}$ & $2.3-3.8 \mathrm{~km}$ \\
RACE & $100 \mathrm{~ms}$ & $0.12-1.2 \mathrm{mV} / \mathrm{m}$ & $20 \mathrm{kHz} / \mathrm{s}$ & mostly up & $1.6-6.5 \mathrm{~s}$ & $1.3-7 \mathrm{~km}$ \\
SIERRA & $70 \mathrm{~ms}$ & $0.02-0.06 \mathrm{mV} / \mathrm{m}^{*}$ & $20 \mathrm{kHz} / \mathrm{s}$ & up/down & $2.4 \mathrm{~s}$ & $3 \mathrm{~km}$ \\
\hline
\end{tabular}

The intensities in the SIERRA experiment are underestimated by up to a factor of 20 due to boom length considerations; see text.

flight; the selected event on the downleg lasts from 685.5 to $689.5 \mathrm{~s}$ corresponding to $585-591 \mathrm{~km}$ altitude. The lower panel shows wave power integrated over the entire $100-\mathrm{kHz}$ displayed frequency range $(1900-2000 \mathrm{kHz})$. The emissions occur on a density gradient, with the strongest waves occurring at $688 \mathrm{~s}$ where $f \approx f_{p e}$. At earlier times, the wave power drops off with time/distance from the matching condition, and the wave frequency is offset from $f_{p e}$ by $0-70 \mathrm{kHz}(0-$ $3.5 \%$ ). In this example, fitting an exponential to the decay yields a decay time of $\sim 2.4 \mathrm{~s}$, corresponding to a decay distance of $3 \mathrm{~km}$.

Table 3 summarizes, by rocket, the relevant characteristics of the waves discussed so far.

These examples demonstrate typical features of the structured Langmuir waves, called "HF-chirps" by McAdams and LaBelle (1999). The waves are not continuous but are composed of many short-lived narrow-band structures with lifetimes of order $50-100 \mathrm{~ms}$. They tend to occur in regions of electron density structure and gradients. The wave power maximizes where $f \approx f_{p e}$. As the rocket moves away from that near-matching condition, into lower-density regions, the waves lie at frequencies further above the local $f_{p e}$ and their amplitude diminishes. The timescale for the decrease in wave power is $0.6-1.1 \mathrm{~s}$ in the two PHAZE II examples analyzed, corresponding to $300-304 \mathrm{~km}, 1.6-6.5 \mathrm{~s}$ in the two RACE examples, corresponding to $745-761 \mathrm{~km}$, and $2.4 \mathrm{~s}$ in the single SIERRA example, corresponding to $585-591 \mathrm{~km}$. Since these experiments included measurements only at a single point in the plasma at each time, it is impossible to determine whether the wave decay time corresponds to a temporal effect or a spatial effect. The slightly shorter timescale in the PHAZE II examples, corresponding to the lowest altitudes, suggests that possibly collisions with neutral particles, which occur at a significantly higher rate at $300 \mathrm{~km}$ versus $580-760 \mathrm{~km}$, may enhance the extinction rate of the waves. However, if the decay times are converted to distances using the rocket velocities, then the two PHAZE II examples correspond to $\Delta h \approx 2.3-3.8 \mathrm{~km}$, the two RACE examples correspond to $\Delta h \approx 1.3-7.0 \mathrm{~km}$, and the SIERRA example corresponds to $\Delta h \approx 3.0 \mathrm{~km}$, so there is in this case no significant difference between the sampled altitude ranges and certainly no systematic increase of $\Delta h$ with altitude. There is also no systematic relation between the decay time and the amount by which the frequency of the waves becomes offset from local $f_{p e}$, which ranges from $2-5.6 \%$ in these experiments.

\section{Conclusions}

In summary, the range of time variations of the structured Langmuir waves observed in these three experiments implies that the term "HF-chirps" is really a misnomer. Only occasionally do the Langmuir wave structures exhibit monotonically decreasing frequency as observed in the PHAZE II experiment by McAdams and LaBelle (1999). More often, other time variations occur, such as monotonically decreasing frequencies, monotonically increasing frequencies, and alternately increasing and decreasing frequencies. However, the $50-100 \mathrm{~ms}$ lifetime of individual fine structure features reported by McAdams and LaBelle (1999) is confirmed by the larger number of observations reported herein. The range of frequency-time slopes of the fine structure features observed during three rocket flights, $0-50 \mathrm{kHz} / \mathrm{s}$, also agrees in magnitude with the range of downward slopes reported by McAdams and LaBelle (1999) based on one flight.

The fine structure emissions follow a characteristic pattern, in which the strongest emissions occur at that point on the electron density gradient where the wave frequency is closest to, but just a little above, the local plasma frequency. As the rocket samples places further away from that condition, the observed wave power decreases. In this paper, we analyzed five examples on three different rocket flights and found that the decay time of the wave power, defined as the e-folding time obtained by fitting the wave decay to an exponential, is in the range $0.6-6.5 \mathrm{~s}$, with the shortest decay times $(0.6-1.1 \mathrm{~s})$ corresponding to the lowest altitudes (300$304 \mathrm{~km})$ and longer decay times (1.3-6.5 s) corresponding to higher altitudes $(585-761 \mathrm{~km})$. However, if the times are converted to distances using the rocket velocities, as would happen if the decaying waves are spatial structures sampled by the rockets, the decay distances average $3 \mathrm{~km}$, and there is no altitude dependence. An altitude dependence might be expected if, for example, the neutral collision frequency plays a major role in determining the extinction rate of the waves. On the other hand it is possible that details such as the amount by which the waves exceed the local plasma frequency matter more than the collision frequency. Detailed 
modelling, beyond the scope of this paper, may be required to interpret these decay time observations. Because the decay times or distances appear fairly consistent across three independent experiments such detailed modelling is warranted.

This paper explores the time variation of the Langmuir wave fine structures, including the frequency variation of individual fine structures, the lifetime of individual fine structures, and the longer decay time or distance associated with the groups of fine structures. Relevant theoretical work, such as work reviewed by Yoon et al. (2006), treats the time stationary case; for example, the eigenmode theory developed by McAdams et al. (2000) and further detailed by Yoon and LaBelle (2005) makes predictions about the number of fine structure features and their frequency spacings, but addresses neither the growth time nor the decay time of these waves. To explain the wave decay requires calculating convective damping rates as the waves propagate in the plasma, experiencing cyclotron and Landau damping. To explain the variations in the frequencies of the fine structures also requires a time dependent model of their generation. The fine structures reported herein bear a superficial resemblance to VLF chorus emissions, for which theoretical explanation has made recent leaps inspired by multi-point measurements made with the Cluster satellites (Inan et al., 2004; Parrot et al., 2003; Santolik et al., 2005). Although these whistler-mode chorus emissions are an entirely different plasma mode, perhaps their generation mechanism provides hints about that of the structured Langmuir waves. In any case, the measurements of fairly consistent fine structure lifetimes and decay distances for the groups of fine structures, observed in three independent auroral rocket experiments, suggest that detailed modelling is warranted.

Acknowledgements. The authors acknowledge teams at NASA Wallops Flight Facility for essential work leading to the success of the three rocket flights used in this study. Co-investigators at Cornell University, University of New Hampshire, University of Alaska-Fairbanks, and University of Iowa were also critical to the success of these experiments. Dartmouth staff engineers M. Trimpi and $\mathrm{H}$. Harjes designed and constructed the HF electric field experiments used in this study. The work at Dartmouth College was supported by NASA grant NNG04WC27G.

Topical Editor M. Pinnock thanks O. Santolik and another referee for their help in evaluating this paper.

\section{References}

Boehm, M.: Waves and static electric fields in the auroral acceleration region, Ph.D. thesis, University of California, Berkeley, 1987.

Ergun, R. E., Carlson, C. W., MacFadden, J. P., Clemmons, J. H., and Boehm, M. H.: Langnuir Wave Growth and Electron Bunching: Results From a Wave-Particle Correlator, J. Geophys. Res., 96, 225-238, 1991a.

Ergun, R. E., Carlson, C. W., MacFadden, J. P., Clemmons, J. H., and Boehm, M. H.: Evidence of Transverse Langmuir Modulational Instability in a Space Plasma, Geophys. Res. Lett., 18, 1177-1180, 1991b.

Gurnett, D. A., Maggs, J. E., Gallagher, D. L., Kurth, W. S., and Scarf, F. L.: Parametric Interaction and Spatial Collapse of beamdriven Langmuir waves in the solar wind, J. Geophys. Res., 86, 8833-8841, 1981.

Inan, U. S., Platino, M., Bell, T. F., Gurnett, D. A., and Pickett, J. S.: Cluster measurements of rapidly moving sources of ELF/VLF chorus, Geophys. Res. Lett., 109, A05214, doi:10.1029/2003JA010289, 2004.

Kintner, P. M., Bonnell, J., Powell, S., Wahlund, J., and Bengt, H.: First results from the Freja HF Snapshot Receiver, Geophys. Res. Lett., 22, 287-290, 1995.

McAdams, K. L. and LaBelle, J.: Narrowband structrure in HF waves above the electron plasma frequency in the auroral ionosphere, Geophys. Res. Lett., 26, 1825-1828, 1999.

McAdams, K. L., Ergun, R. E., and LaBelle, J.: HF chirps: Eigenmode trapping in density depletions, Geophys. Res. Lett., 27, 321-324, 2000.

McFadden, J. P., Carlson, C. W., and Boehm, M. H.: Field-aligned electron precipitation at the edge of an arc, J. Geophys. Res., 91, 1723-1730, 1986.

Parrot, M., Santolik, O., Cornilleau-Wehrlin, N., Maksimovic, M., and Harvey, C. C.: Source location of chorus emissions observed by Cluster, Ann. Geophys., 21, 473-480, 2003, http://www.ann-geophys.net/21/473/2003/.

Samara, M.: Sounding Rocket Investigations of Whistler, Upper Hybrid and Langmuir Waves in the Auroral Ionosphere, Ph.D thesis, Dartmouth College, Hanover, NH, 2005.

Santolik, O., Gurnett, D. A., Pickett, J. S., Parrot, M., and Cornilleau- Wehrlin, N.: Central position of the source region of storm-time, chorus, Planet. Space Sci., 53, 299-305, 2005.

Temerin, M.: The polarization, frequency, and wavelengths of highlatitude turbulence, J. Geophys. Res., 83, 2609-2616, 1978.

Yoon, P. H. and LaBelle, J.: Discrete Langmuir waves in density structure, J. Geophys. Res., 110, A11308, doi:10.1029/2005JA011186, 2005.

Yoon, P. H., LaBelle, J., Weatherwax, A. T., and Samara, M.: Mode Conversion Radiation in the Terrestrial Ionosphere and Magnetosphere, in: Geospace Electromagnetic Waves and Radiation, edited by: LaBelle, J. W. and Treumann, R. A., Springer, Heidelberg Edn., p. 211, 2006. 